Virginia Commonwealth University vCU Scholars Compass

\title{
Effect of Field Direction on Electrowetting in a Nanopore
}

D. Bratko

Virginia Commonwealth University, dbratko@vcu.edu

Christopher D. Daub

Virginia Commonwealth University

Kevin Leung

Sandia National Lab

Alenka Luzar

Virginia Commonwealth University

Follow this and additional works at: https://scholarscompass.vcu.edu/chem_pubs

Part of the Chemistry Commons

(C) 2007 American Chemical Society

\section{Downloaded from}

https://scholarscompass.vcu.edu/chem_pubs/95

This Article is brought to you for free and open access by the Dept. of Chemistry at VCU Scholars Compass. It has been accepted for inclusion in Chemistry Publications by an authorized administrator of VCU Scholars Compass. For more information, please contact libcompass@vcu.edu. 


\title{
Effect of field direction on electro-wetting in a nanopore
}

\author{
Dusan Bratko ${ }^{1,2, a, *}$, Christopher D. Daub ${ }^{1}$, Kevin Leung $^{3}$ and Alenka Luzar ${ }^{1, \mathrm{~b}, *}$ \\ ${ }^{1}$ Department of Chemistry, Virginia Commonwealth University, Richmond, VA 23284-2006 \\ ${ }^{2}$ Department of Chemical Engineering, University of California, Berkeley, CA 94720-1462 \\ ${ }^{3}$ Sandia National Laboratories, MS 1421, Albuquerque, NM 87185 \\ a: dnb@berkeley.edu, b: aluzar@vcu.edu
}

December 16, 2006

\begin{abstract}
We manifest a significant influence of field direction and polarity on surface wetting, when the latter is tuned by application of an external electric field. Thermodynamics of field-induced filling of hydrocarbon-like nanopores with water is studied by open ensemble molecular simulation. Increased field strength consistently results in waterfilling and electrostriction in hydrophobic nanopore. A threshold field commensurate with surface charge density of about one elementary charge per $10 \mathrm{~nm}^{2}$ suffices to render prototypical paraffin surfaces hydrophilic. When field is applied in the direction perpendicular to the confining walls, the competition between orientational polarization and angle preferences of interfacial water molecules relative to the walls results in asymmetric wettability of opposing surfaces (Janus interface). Reduction of surface free energy observed upon alignment of confinement walls with field direction suggests a novel mechanism whereby the applied electric field can operate selectively on waterfilled nanotubes while empty ones remain unaffected.
\end{abstract}




\section{INTRODUCTION}

Most hydrophilic solutes derive their affinity for water from attractive interactions between ionized or polar groups of the solute with dipoles and quadrupoles of water molecules ${ }^{1,2}$. Application of external voltage across a solid/liquid interface can produce a similar effect as water dipoles interact favorably with the external field reducing interfacial free energy. This phenomenon is exploited in a variety of applications from microfluidics to electrospray ionization and ink-jet printing ${ }^{3-6}$ to electrical control of optical devices ${ }^{7,8}$. The advent of microand nanoporous materials and nanotubes ${ }^{9,10}$ sparked renewed interest in wetting techniques including electro-wetting ${ }^{11,12}$, as high surface-to-volume ratio makes these media especially difficult to permeate with water. Electro-wetting has also been discussed in context of water permeation through ion channels ${ }^{13}$.

Macroscopic electro-capillarity experiments are relatively well described within continuum approximations where water is treated as a structureless medium of constant permittivity $\varepsilon=\varepsilon_{r} \varepsilon_{0}$. Macroscopic relations pertinent to electrowetting in a planar confinement (Section S.I. in Supporting Information) predict reduction in (cosine of) contact angle, increase in pressure and density, and reduction in threshold pore-width of capillary evaporation to be proportional to the applied electric field squared. Assumptions underlying the continuum picture are, however, no longer valid at nanoscale where molecular structure of the liquid, including orientational preferences of surface molecules ${ }^{14,15}$, becomes important and the fraction of molecules in the boundary layer of the liquid represent an increasingly significant constituency ${ }^{16}$. Further, the macroscopic concept of surface energies and surface forces becomes ambiguous at molecular resolution. 
To overcome experimental and conceptual limitations that preclude predicting surface tension or contact angle in the nanoscale regime, and to extract molecular level insights from the response of the interfacial-liquid to the external field, we use molecular simulations. We consider a broad range of electric fields including typical field strengths in ion-channels and membranes $^{17,18}, \quad 10^{-2} \mathrm{~V} \AA^{-1} \leq E_{o} \leq 0.4 \quad \mathrm{~V}^{-1}\left[E \sim \mathrm{O}\left(10^{-2}\right) \quad \mathrm{V}^{-1}\right]$. Here, $\quad E$ denotes the actual (dielectrically screened) field and $E_{o}$ the applied field inside an empty confinement. The above field strengths suffice to secure a noticeable alignment of aqueous molecules with the field, revealing the heretofore-neglected influence of field direction relative to liquid surfaces. This allows us to study the combined field effect comprising

a) the "bulk" term (increasing with the volume of confined water interacting with the field), which is the primary cause of field-assisted spreading of water into confinement, and

b) pure surface effects reflecting the coupling between the orientational bias for molecules at interfaces and molecular alignment along the direction of electric field.

The latter phenomenon is reflected in notable surface tension dependence on the direction of electric field as demonstrated by our simulations. Atomistic signatures such as the orientation of water dipoles at surfaces are examined, and they can potentially be compared with second harmonic generation experiments.

A number of molecular studies concerned with the role of electric field on thermodynamics, structure and dielectric behavior of water in nano-sized apolar confinements have been reported recently ${ }^{13,19-23}$. Electric field applied parallel to the solid surface invariably increased the wettability of the confinement. Recent simulation of water confined between mesoscopic graphene platelets immersed in a bulk, field-free reservoir, however, showed weakly asymmetric water density depletion in the intervening region upon application of a perpendicular 
electric field between the plates ${ }^{22}$. This contradicts the qualitative predictions of continuum theories (Summarized in Section S.I. of Supporting Information). Our systematic results confirm a general increase in water density in confinement under exposure to electric field.

Neither of the reported studies paid explicit attention to the possible role of field direction. The observed ${ }^{20,22,24}$ alignment of water molecules in the direction of the applied field, however, can couple ${ }^{22}$ with known anisotropy ${ }^{14,15,25-29}$ of interfacial water. This suggests that the angle between the direction of the applied field and water/solid surfaces can also play a significant role in field-induced wetting of nanoscaled hydrophobic confinements. To elucidate the energetics of the phenomenon on the molecular level, we use open ensemble simulations of water in apolar confinement under varied electric fields, maintaining equilibrium with field-free bulk reservoir of water. We determine the apparent wall/liquid interfacial free energy change as a function of the strength of external field and its direction and compare the observed effects with (smaller) changes seen at the water/vapor interface at similar conditions. While model pore material is strongly hydrophobic, with simulated contact angle $\theta_{c}$ close to $135^{\circ}{ }^{30}$, we find that spontaneous evaporation, observed $\mathrm{d}^{30-33}$ in narrow confinements in the absence of the field, can be prevented when the electric field is applied. Interestingly, electrostriction is stronger and crossover to wetting behavior is observed at weaker field strength when the field is directed laterally along the confinement walls. In a perpendicular field, the wetting ability differs for the two walls as field-preferred molecular orientations relative to the solid surfaces at opposite walls differ by $180^{\circ}$. By carefully tuning the field strength, the positively charged wall can turn hydrophilic while the other wall remains hydrophobic, a behavior associated with a Janus interface $e^{34}$. The observed influence of field direction is consistent with orientational preferences that maximize water hydrogen bonding. 


\section{MODEL SPECIFICATIONS}

Our model system consists of a pair of parallel hydrophobic plates separated by distance $D$, varying within the range from 0.9 to $\sim 4 \mathrm{~nm}$. Intervening water is described using the extended simple point charge model $(\mathrm{SPC} / \mathrm{E})^{35}$. Because we are interested in the possibility of electro-wetting in strongly hydrophobic systems, conducive to capillary evaporation in field-free $\operatorname{cases}^{30,33}$, we describe the interaction of a water molecule with either of the walls, $u_{w}(z)$, by the integrated Lennard-Jones (9-3) potential with parameters roughly corresponding to the hydrocarbon-water potential ${ }^{14,15,30,33}\left(\theta_{c}=135 \pm 5^{\circ}{ }^{30}\right)$. A detailed description of surface-water potential and pertinent interaction parameters are given in Section S.II of Supporting Information.

As shown in previous works, the given wall/water interaction leads to spontaneous waterto-vapor transition in the slit when $D$ is sufficiently small ${ }^{30,33}$. For given (hydrophobic) wall material, evaporation is kinetically viable over accessible simulation times $\left[\mathrm{O}\left(10^{7}\right)\right.$ passes] for $D$ below $\sim 1.3 \mathrm{~nm}$, slightly above three monolayers of water. Metastable liquid persists in initially water-filled slits at bigger separations $\mathrm{s}^{33,36-38}$. Capillary evaporation ${ }^{39}$ precludes systematic studies in narrower pores. The present system is comparatively more conducive to water depletion than is the graphite-like confinement (contact angle close to $90^{\circ}$ ) studied in ref. ${ }^{22}$ and is well suited to verify the occurrence of eventual density depression in an electric field observed in that work ${ }^{22}$.

In addition to intermolecular and water/wall interactions, the system Hamiltonian includes interactions between the partial charges on water molecules ${ }^{35}$ and the external field $\vec{E}=-\nabla \psi$, where $\psi$ is the electrostatic potential. Since we only consider fields along direction $z$, perpendicular to confinement walls, or $x$, parallel to the walls, we use a simplified notation $E_{\perp}$ and $E_{\|}$to denote the field along either of the two directions. Listed values of $E_{\perp}$ or $E_{\|}$pertain 
to the applied electric field $0 \leq\left|E_{o}\right| \leq 0.4 \mathrm{VA}^{-1}$ (without the contribution from water polarization).

The median value of the above range of field strengths, $0.2 \mathrm{VA}^{-1}$, corresponds to the unscreened field next to an extended electrified interface with surface charge density of one elementary (proton) charge per $9 \mathrm{~nm}^{2}$ and is about an order of magnitude weaker than unscreened fields around common ions. Our calculations to this strong field regime complement previous studies of related effects in the bulk $k^{24,40,41}$ and at interfaces ${ }^{19-23,42}$ by pertinent wetting energetics; to establish connection with these works requires including fields that are of comparable, or at least not essentially smaller strengths than those used in refs ${ }^{13,19-24,40}$. While it is useful to specify model conditions in terms of unscreened field $E_{o}$, the actual electric field, $E$, is vastly reduced through the polarization of the medium, i.e. water ${ }^{20,43}$, varying within $0 \leq|E| \leq \sim 0.04 V \AA^{-1}$. To put this in better perspective, we note only local fields exceeding $\sim 1 V \AA^{-1}$ have been found ${ }^{44}$ capable to visibly polarize aqueous molecules in simulations using a polarizable force field for water $^{45}$.

Other model details are the same as described earlier ${ }^{33,46}$. Focusing on the qualitative picture of the system, apart from a few test calculations employing two-dimensional lattice sums in lateral directions to match Ewald sum results, we use lateral periodic conditions with water/water interactions subject to a smooth spherical cutoff; the form of the cutoff was the same as given in ref. ${ }^{15}$ where this choice has been rationalized by systematic comparison with alternative types of periodic conditions. The smooth cutoff takes place between 2.96 and 3.16 diameters of a water molecule, making the range of interaction essentially identical to that employed in water contact angle calculations in droplet geometry ${ }^{47}$. No truncation is applied to water/wall interactions. 


\section{SIMULATION}

To describe the equilibrium between confined water exposed to electric field, and fieldfree bulk phase, we use Grand Canonical Monte Carlo algorithm described earlier ${ }^{31,33,46}$. Configurations are therefore generated using Metropolis acceptance criteria for molecular moves, additions and deletions of molecules, and collective moves of confined water slab relative to the walls ${ }^{33}$. The thermodynamic state of bulk SPC/E water was determined by setting excess chemical potential $\mu_{e x} / k_{B} T=-12.1$, a value chosen to obtain bulk pressure $P=0 \pm 15$ atm, as determined in separate simulations for the bulk phase. Temperature $\mathrm{T}=298 \mathrm{~K}$ was presumed in all cases.

Typical system dimensions $L_{x y}$ and $L_{z}=D$ used in simulation calculations of interfacial free energy, $\sigma$, were 2.1 and $1.64-2.7 \mathrm{~nm}$, respectively. This corresponded to between 160-200 water molecules at the smaller, and 310-350 molecules at the larger wall separation; the number of molecules varied with the strength of the applied electric field and concomitant electrostriction. To secure numerical accuracy in $\sigma$ of $\pm 3 \mathrm{mN} \mathrm{m}^{-1}$, individual runs required $\mathrm{O}\left(10^{9}\right)$ attempted configurations, primarily due to low acceptances of particle insertions. To keep computations within practical limits, spherical cutoff combined with laterally periodic conditions was employed in most of the calculations, while we used the MMM2 $\mathrm{D}^{48}$ method to calculate the two-dimensional lattice sum (essentially equivalent to Ewald $\operatorname{sum}^{49}$ ) in a limited number of runs to evaluate possible finite-size effects associated with selected system size and boundary conditions.

\section{NEW APPROACH TO WETTING FREE ENERGIES IN A NANOPORE}

The work associated with spreading of bulk water (at constant chemical potential $\mu$, and temperature $T$ ), into a planar confinement with given wall-wall distance $D$ is best characterized in 
terms of the grand potential $\Omega\left(A_{s l}\right)$, where $A_{s l}$ is the solid/liquid contact area (comprising wet areas on both confinement walls), and $V=A_{s l} D / 2$ is the liquid-occupied volume in the confinement. The change in $\Omega$ corresponding to an increase in the contact area of $\mathrm{d} A_{s l}$ can be determined as the sum of changes associated with two steps: 1) water slab deformation increasing the wetted area $A_{s l}$ by $\mathrm{d} A_{s l}$ at constant volume. Conservation of volume implies simultaneous reduction in $D$ such that $\operatorname{d} \ln D=-\operatorname{d} \ln A_{s l}$, and 2) subsequent increase in $D$ restoring the original slit width. During the second step, $A_{s l}$ is kept constant. In the first step, performed at fixed volume and amount of the liquid, $\mathrm{d} \Omega_{1}=-\frac{1}{2}\left(P_{\perp} A_{s l} d D+P_{\|} D d A_{s l}\right)=\frac{D}{2}\left(P_{\perp}-P_{\|}\right) d A_{s l}=\Delta \gamma^{\prime} d A_{s l}$, where we include contributions from surface (water/wall) forces in $P_{\alpha \beta}$ and $\Delta \gamma^{\prime}$. $\Delta \gamma^{\prime}=\left(P_{\perp}-P_{\|}\right) d / 2$ is replacing $\Delta \gamma=\gamma_{s l}-\gamma_{s v}$ in a process where increase in contact area is associated with reduction in confinement wall-wall separation $D$. During the second step, a small amount of the liquid is allowed to enter the pore as pore width $D$ is returned to the initial value while $A_{s l}$ is kept constant. During this step, volume is increased by $\mathrm{d} V=\mathrm{d} A * D / 2$ and $\mathrm{d} \Omega_{2}=-\left(P_{\perp}-P_{b}\right) \mathrm{d} A^{*} D / 2$. For ambient pressure, $P_{b}=\mathrm{O}(1 \mathrm{~atm})$, volume work on the bulk phase can be neglected. No work is associated with mass transfer between the bulk $(b)$ and confined $(c)$ phases because of equal chemical potentials, $\mu_{b}=\mu_{c}=\mu$. Work per unit area of the solid/liquid interface, $\sigma$, is:

$$
\sigma=\frac{\partial \Omega}{\partial A}=\frac{\partial \Omega_{1}}{\partial A}+\frac{\partial \Omega_{2}}{\partial A}=\Delta \gamma^{\prime}-\frac{P_{\perp} D}{2}=-\frac{P_{\|} D}{2}
$$

and $P_{\|}$denotes the slab average of $P_{\|}(z)$ where $\mathrm{z}$ is the distance from pore mid-plane. When an electric field is present, $\sigma$ also includes electrical work, $W_{e l}$, (See Section S.I of Supporting Information) which pertains to the total slab and not to the surfaces alone. Note that, for systems 
of finite width, $\Delta \gamma^{\prime}$ also includes the contribution of solvation pressure acting between confinement walls. At nanoscale separations, both $\Delta \gamma^{\prime}$ and $P_{\perp}$ depend on $D$. The apparent interfacial tension $\sigma$, corresponding to water spreading into the confinement at fixed separation $D$, on the other hand, can be almost independent of $D$ when the electric field is turned off. In field-free systems, for large wall-wall separations, $P_{\perp} \rightarrow 0$, with $\sigma$ and $\Delta \gamma^{\prime}$ both approaching the single-wall value $\Delta \gamma=\gamma_{s l}-\gamma_{s v}$.

To study the variation of water surface tension, $\gamma_{l}$, under the influence of the field, we also performed closed ensemble ( $N V T$ ) simulations of an unconfined water slab occupying a layer along the midplane of the simulation cell. The initial slab configuration was prepared in a field-free confined system of widths between 2.7 and $4 \mathrm{~nm}$. In subsequent simulation, no walls were present and the liquid was in contact with its own vapor filling the remainder of the cell; hence the standard relation $\gamma_{v}=\left(P_{\perp}-P_{\|}\right) D / 2$ was employed in these cases. Calculation of pressure tensor components is described in Section S.I. of the Supporting Information.

\section{RESULTS AND DISCUSSION}

\section{V.1 Electrostriction in a narrow confinement}

According to macroscopic thermodynamics, electrostriction or increase in liquid density in applied electric field is proportional to the compressibility of the liquid (eq S.3 in Supporting Information). As compressibility of liquid water, $\kappa \sim 4.6 \cdot 10^{-4} \mathrm{MPa}^{-1}$, is very low, we observe only small density changes of $\mathrm{O}(1 \%)$ in the bulk aqueous phase in the strongest field we consider. Water adjacent to non-polar interfaces, however, behaves very differently. With local density of water in the immediate vicinity of a hydrophobic surface slightly depleted, the liquid becomes 
much more compressible $\mathrm{e}^{33,50-52}$. Under electric field, higher compressibility translates into stronger electrostriction. In Fig. 1 we present our results for relative increase in average water density in a planar pore for two widths, $D=1.64$ and $2.7 \mathrm{~nm}$ when the system is placed into external fields of strengths $0 \leq\left|E_{0}\right| \leq 0.4 \mathrm{VA}^{-1}$ with field directions perpendicular or parallel to the walls. Quite surprisingly, electrostriction is much stronger in the parallel field, an interesting and new observation to which we will return shortly.

The inset in Fig. 1 shows the field strength dependence of the reduced compressibility $\kappa_{r}=\rho k T \kappa$, determined from number density fluctuations: $\kappa_{r}=\left(\left\langle N^{2}\right\rangle-\langle N\rangle^{2}\right) /<N>$, in a pore with $\mathrm{D}=2.7 \mathrm{~nm}$. High compressibility of confined water can be attributed to increased fluctuations in number densities in the immediate vicinity of the walls. As field strength is increased, these

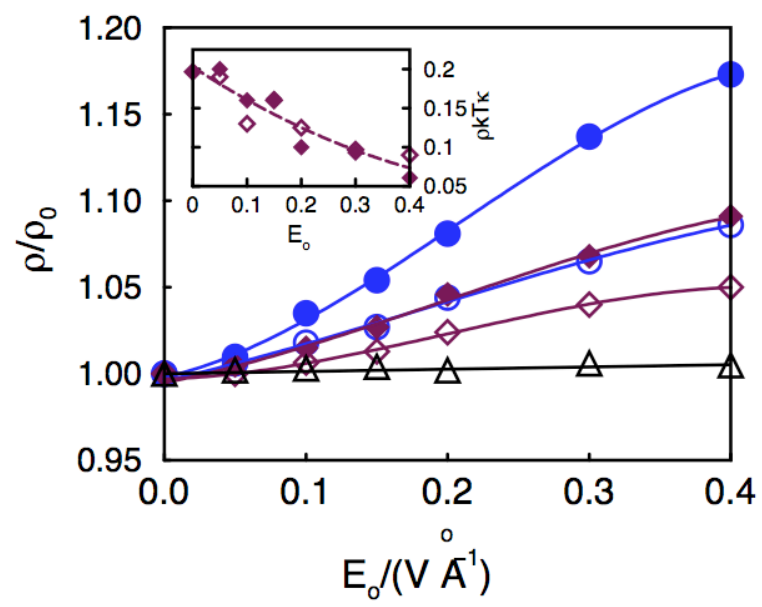

Figure 1 Relative increase in average liquid density as a function of applied electric field $E_{o}$ in bulk water (black) and hydrocarbon-like confinements of width $1.64 \mathrm{~nm}$ (blue) or $2.7 \mathrm{~nm}$ (magenta) for perpendicular (open) or parallel (filled symbols) direction of the field. Inset: Reduced isothermal compressibilities of water, $\kappa_{r}=\rho k T \kappa$, in the $2.7 \mathrm{~nm}$ confinement for field applied in parallel (filled) or perpendicular direction (empty symbols). Bulk reduced compressibility of water is $\sim 0.065$. 
fluctuations become suppressed as liquid is packed into the pore. This explains the saturation trend observed in the main graph at strong fields $E_{o}$.

Compared to bulk electrostriction, the relative density increase for two confinement sizes is quite dramatic. The $\sim 13 \%$ density increase in the $1.64 \mathrm{~nm}$ confinement with parallel field $E_{o}=0.28 \mathrm{~V}^{-1}$ conforms with $12 \%$ density increase in identical field inside a cylindrical ion channel of width $1.4 \mathrm{~nm}$, the reported system $^{20}$ in the literature most similar to any of our cases. Our data also agree qualitatively with increased prevalence of water-filled states for nanotubes exposed to fields of similar strength ${ }^{21}$. Although we use strongly hydrophobic walls conducive to water expulsion $^{33}$, we observe no instances of field-induced density depression such as reported recently $^{22}$ for planar graphene confinements under the field applied across the confinement. Moreover, for the hydrocarbon confinement we consider, we show that the applied field can prevent capillary evaporation at small inter-plate separations below kinetic threshold distance ${ }^{33,36}$ (threshold of spinodal decomposition) of about $1.3 \mathrm{~nm}$ where water consistently evaporates in the absence of the field. For example, for field strengths $E_{o}=0.12,0.24$, and $0.36 \mathrm{~V} \AA^{-1}$, evaporation is suppressed by the field at separations $D \geq 0.12,0.11$, and $0.092 \mathrm{~nm}$, respectively. In our model calculations for a variety of conditions, electrostriction and field-stabilization of liquid state (relative to vapor), both known in bulk water generally apply and are reinforced in the confinement ${ }^{53}$. A number of theoretical studies reported on electrofreezing in bulk and confined aqueous phases ${ }^{24,40-42,54-58}$. In these works, the formation of simulated ice was observed either at higher field strengths ${ }^{55,56}$ or lower temperatures ${ }^{24,40}$ than used here, or was assisted by nucleation on strongly polar confinement walls ${ }^{42,57,58}$. No instances of freezing have been observed in our model system at ambient temperature, comparatively weak fields and strongly hydrophobic (hydrocarbon-like) confinement. 


\section{Pressure variation in electric field}

Upon an increase in the strength of the field the amount of confined liquid (in equilibrium with field-free bulk phase) will increase until the elevated pressure balances chemical potentials inside and outside of the confinement. In Fig. 2, we show the observed field-induced change in the pressure on the confinement walls, $P_{z z} \equiv P_{\perp}$, at two confinement widths, and compare it to the pressure in field-exposed bulk phase, all at fixed chemical potential. In accordance with macroscopic predictions, pressure monotonically increases withthe field.

Density and orientation profiles of water in the confinement in electric field

A very interesting feature emerges upon comparison between electrostriction results for fields of different directions. As shown in Fig. 1, in the narrow confinements we study, the overall electrostriction is much stronger when the field is aligned with confinement walls than for perpendicular fields of equal strength. Because of its asymmetry and anisotropic hydrogen

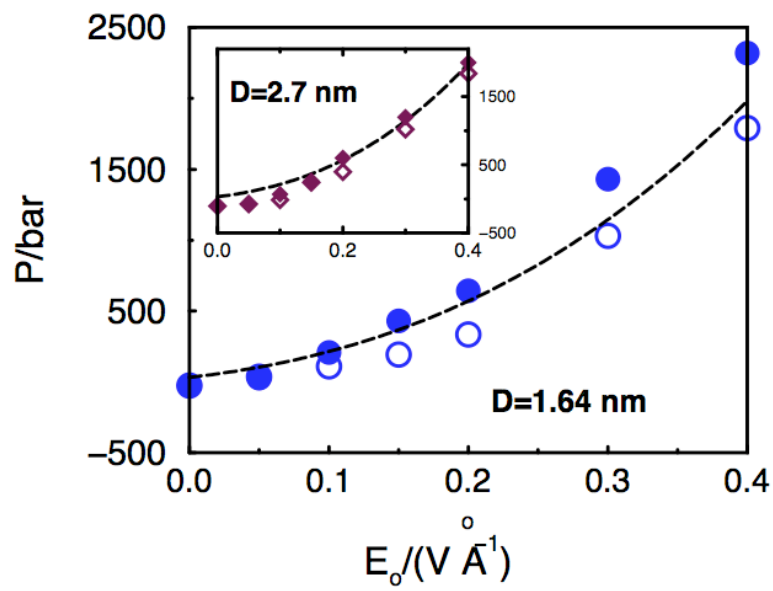

Figure 2 Pressure increase with increasing strength of applied electric field in bulk aqueous phase (dashed line) or in a planar confinement of varied widths $D$ as a function of the strength of the electric field applied in directions parallel (solid) or perpendicular (open symbols) to confinement walls. 
bond interactions, water has long been known for orientation bias at interfaces ${ }^{27}$ with approximately parallel dipole alignment favored ${ }^{14,15,28}$ as it minimizes the loss of hydrogen bonds on the surface. This bias exists only in the surface layer, hence the influence of the direction of the field becomes relatively more important as the pore width is reduced and the fraction of surface molecules gets larger.

For perpendicular field, the molecular asymmetry and orientational preferences of surface molecules result in distinction between incoming and outgoing field. This means that structures and energetics at the two surfaces, symmetric in the absence of the field, will differ when the field is sufficiently strong to align water molecules noticeably in its direction, i.e. the product $E \mu(\mu$ is the dipole moment of water $)$ is not much smaller than the thermal energy $k_{B} T^{22}$. At ambient conditions this implies $E \geq \sim 5 \cdot 10^{-3} \mathrm{~V}^{\circ} A^{-1}\left(E_{o} \sim E \varepsilon=\mathrm{O}\left(0.1 \mathrm{~V} \stackrel{\circ}{A}^{-1}\right)\right.$. In Fig. 3a, we compare density profiles of water oxygens across a pore of width $D=2.7 \mathrm{~nm}$ at zero field and for fields of strength $E_{o}=0.2 \mathrm{~V}^{-1}$ applied in parallel and perpendicular directions. In parallel field, wetting is enhanced at both walls. Contact layer peaks in density profiles are strongly increased and shifted closer to the walls consistent with increased pressure in the pore. In perpendicular field, however, the profile is very asymmetric, with strongly enhanced wetting on the left wall, where the field orients water oxygens toward the wall and hydrogens into the liquid phase. Despite the strongly elevated pressure in the confinement $\left(P \sim 5 \cdot 10^{2}\right.$ bar $)$, water density on the opposite wall remains almost unchanged. In the absence of electric field, orientational bias emerges as interfacial water molecules tend to optimize hydrogen bonding ${ }^{14,15,27,28}$. The interplay between this effect and alignment with electric field results in the asymmetric wettability at opposing walls in the perpendicular field. Within a range of field strengths, including the situation depicted 
in Fig. 3a, the properties of opposing surfaces can be tuned to form the so called Janus interface ${ }^{59}$ consisting of parallel surfaces of opposite, hydrophilic and hydrophobic characters.

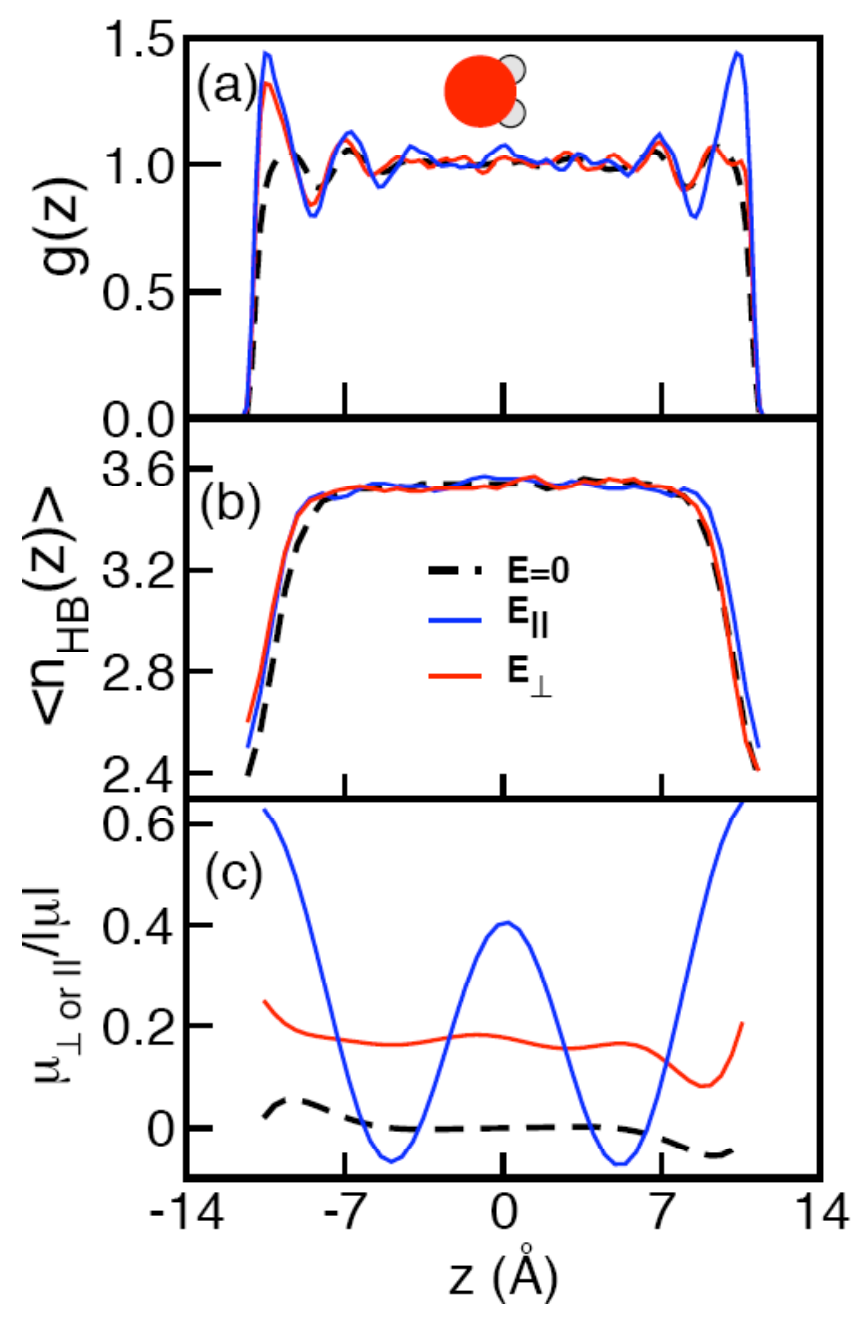

Figure 3 (a) Density profile of confined water in a hydrocarbon-like confinement of width $D=2.7 \mathrm{~nm}$ in the absence of electric field (black), and in perpendicular (red) and parallel (blue lines) field of strength $0.2 V^{-1}$, with the orientation of water molecules favored by the applied perpendicular field. $3(\mathbf{b})$ Average number of hydrogen bonds per molecule of water, $\left\langle n_{H B}\right\rangle$, as a function of the distance from pore midplane, $z .3$ (c) Orientational polarization of aqueous molecules measured in terms of the average dipole component in the direction of applied field $E_{0}=0$ or $0.2 V \stackrel{o}{-1}, \mu_{\perp} /|\mu|$ or $\mu_{\|} /|\mu|$ as a function of molecular position relative to the walls. Included is spontaneous polarization normal to walls in the absence of external field. 
To verify the connection with hydrogen bonding, in Fig. $3 \mathrm{~b}$ we show profiles of average numbers of hydrogen bonds per water molecule, $\left\langle n_{H B}(z)>\right.$ for confined water in the absence of the electric field and for perpendicular or parallel fields of strength $0.2 \mathrm{~V} \AA^{-1}$. On the whole, hydrogen bonding is somewhat enhanced in the presence of the field in agreement with predictions from a recent theoretical analysis ${ }^{41}$. For perpendicular field, our results reveal a notable increase in hydrogen bonding at the (positively charged) wall, at which the field brings oxygen atoms closer, and hydrogens further from the walls. The difference of $\sim 0.25$ hydrogen bonds corresponds to an average energy difference of close to $2 \mathrm{kT}$ per molecule for molecules at opposite walls. In the perpendicular electric field, only wetting on one of the walls is significantly enhanced; the overall density increase in the parallel field is therefore almost twice that observed in perpendicular field of equal strength.

While the incentive for wetting derives from field interactions with molecules at the surfaces and in the slab interior ${ }^{16}$, only surface molecules are subject to the sign preference discussed above. Accordingly, the discrimination with respect to field direction should not depend on the width of the confinement, an expectation borne out by our results for two pore widths (Fig. 4a). From a practical point of view, the field direction should be regarded as one of the determinant factors in designing electro-wetting techniques in nanopores where interfacial molecules represent a significant fraction of the total amount of the liquid.

\section{V.2 Surface energetics}

\section{V.2.1 Energy dependence on the applied field}

In agreement with structural results, average energies per molecule (available in Section S.IV of Supporting Information), are significantly lower in nanopores in which the direction of 
electrical field is parallel with the walls. Hydrogen bonding profiles presented in Fig. 3b confirm that parallel water-wall alignment preserves a high fraction of hydrogen bonds and can do so without competing with the effects of applied field. In perpendicular fields, as a result of fieldinduced shift in orientational distributions, hydrogen bonding is enhanced on one wall but somewhat weakened on the other. These results show there is significant anisotropy in molecular responses to the applied field ${ }^{43}$ at the walls. Clearly, the parallel component of the interfacial liquid permittivity, $\varepsilon_{\|}$will exceed the average value of the normal component, $\varepsilon_{\perp}$. Because of orientational restrictions imposed by hydrogen-bonding, the normal component will depend on field polarity. Significant reduction in $\varepsilon_{\perp}$ is expected for field direction orienting aqueous dipoles toward the adjacent confinement wall. Simulated polarization profiles shown in Fig. $3 \mathrm{c}$ confirm this expectation.

\section{V.2.2 Interfacial tensions in electric field}

To characterize our model system and test the algorithms, we first performed simulations for field-free aqueous slabs and confinements. These reference data are collected in section S.V of Supporting Information. All results for field-exposed systems are presented in Fig. 4. The top two curves in Fig 4a illustrate the effect of the field on water surface tension (squares) calculated for a semi-infinite liquid slab of thickness corresponding to approximately seven layers of water molecules, a width chosen to match the thickness of the water film in the wider of the two confined geometries we consider. To preserve the free liquid/vapor interface, the sampling for this geometry had to be carried out within a closed $(N, V, T)$ ensemble. Any increase in the area of the liquid/vapor interface takes place as a result of slab thinning at constant volume. Here, the 
system comprises coexisting vapor and liquid, however both phases are exposed to the field and the chemical potential will deviate from its value in the field-free bulk phase.

In both normal and parallel fields, liquid/vapor surface tension $\gamma_{l v}$ decreases with increasing field strength. This decrease (only weakly dependent on field direction) is interpreted as resulting from comparatively stronger interaction of water dipoles with the applied field at liquid/vapor surfaces ${ }^{43}$.

Our main results are described by the remaining four curves in Fig. 4a showing solid/liquid wetting surface free energies (eq 1) for both the narrower $(D=1.64 \mathrm{~nm}$, circles) and wider ( $D=2.7 \mathrm{~nm}$, diamonds) confinements in perpendicular (open symbols) and parallel field (solid symbols). Compared to liquid/vapor surface tensions wetting of the solid confinement reveals much stronger field dependence. Here, water spreading on the surfaces is associated with transfer from the field-free bulk phase into the field-exposed confinement. Unlike the process of slab deformation used in the calculation of $\gamma_{l v}$, where the amount of water exposed to the field is constant, here the energetic incentive for wetting comes from the increase in the number of water molecules interacting with the field. Since the field permeates the entire aqueous slab, $\sigma$ comprises both surface and volume effects and the influence of the field is stronger at increased $D$. Change in sign of $\sigma$ signifies the field-induced transition from drying to wetting behavior. In the inset of Fig. 4a, showing the crossover region, $\sigma$ is presented as a function of the surface charge density, $q_{\mathrm{s}}$, capable of producing the given unscreened field strength $E_{o}$. For paraffin-like wall material considered in our examples, the minimum interfacial charge densities that suffice to switch model walls from hydrophobic $(\sigma>0)$ to hydrophilic $(\sigma<0)$ vary over the range corresponding to one elementary charge, $e_{o}$, per $\sim 7.6-12.5 \mathrm{~nm}^{2}$. 

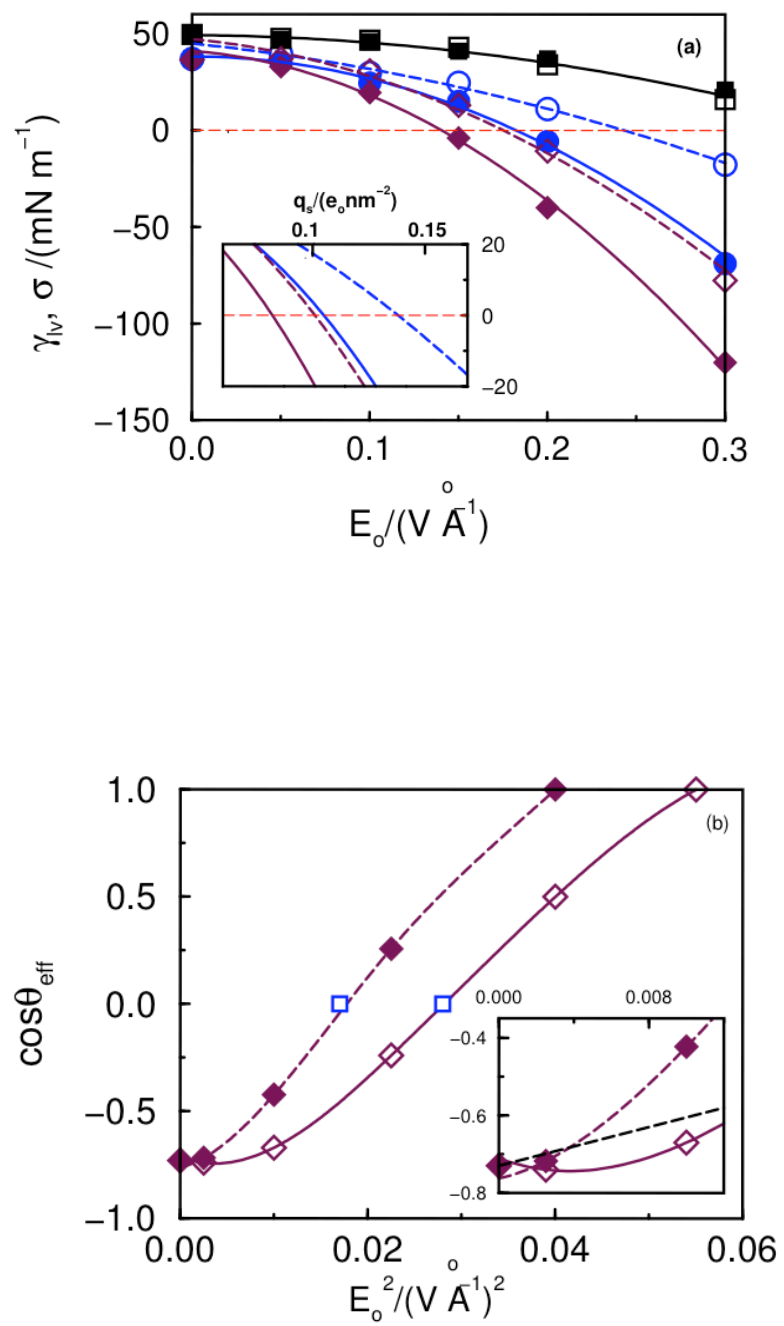

Figure 4 (a) Surface tension, $\gamma_{l v}$, of water in semi-infinite aqueous slab of width corresponding to approximately seven molecular layers in contact with vapor (squares), or wetting surface free energy of hydrocarbon/water interface, $\sigma$, in confinement of width $1.64 \mathrm{~nm}$ (circles) or $2.7 \mathrm{~nm}$ (diamonds) as a function of the strength of electric field, $E_{o}$, applied in perpendicular (empty) or parallel direction (solid symbols) relative to the surfaces. Red dotted lines separate drying (top) and wetting (bottom) regimes. The inset shows surface charge densities, $q_{s}$, (in units of elementary charge, $e_{o}$, per $\mathrm{nm}^{2}$ ) corresponding to unscreened field strengths $E_{o}, 4$ (b) Hypothetical contact angle $\cos \theta_{\text {eff }}=-\sigma / \gamma_{l v}$, illustrating wetting energetics in parallel (solid) or perpendicular (empty symbols) electric field in a hydrocarbon-like confinement. Blue squares correspond to $\sigma=0$. Inset: comparison of simulation results with low-field prediction (black dashed line). 
The strong dependence of $\sigma$ on field direction, revealed by significant differences between the results for the perpendicular and parallel fields (Fig. 4), demonstrates the importance of surface layer effects. Although the net field effect is stronger in a wider pore, the role of field direction is relatively more important in the narrower of the two confinements.

As shown in Fig. 3, when the electric field is perpendicular to the walls (Fig. 3a), surface structure and thermodynamics on individual walls strongly depend on field polarity. The data in Fig. 4a, showing the influence of field strength on $\sigma$ in the perpendicular field, pertain to the two-wall average directly accessible from simulation. The average $\sigma$ corresponds to work associated with simultaneous wetting of both confinement walls as the pore is filled with water. Confinement wettability can be formally characterized in terms of thermodynamic contact angle $^{60} \theta_{e f f}$, with $\cos \theta_{e f f}=-\sigma / \gamma_{l v}$. Based on macroscopic predictions ( eqs S.1-2 of Supporting Information), when the dependence of $\gamma_{v}$ on $E$ is weak, $\cos \theta_{\text {eff }}$ should vary approximately linearly with field squared. Fig. $4 \mathrm{~b}$ compares the dependences of $\cos \theta_{\text {eff }}$ on $E^{2}$ for the two field directions. Here, we approximated $\gamma_{l v}$ by the value calculated for electric field of given strength and direction normal to that experienced by the walls. The inset in Fig. $4 \mathrm{~b}$ shows the initial slope of the curve $\cos \theta_{\text {eff }}\left(E^{2}\right)$ for the parallel field to be close to the weak field prediction. Smaller initial slope in perpendicular field reflects the conflict between optimal water molecule/wall orientation and alignment with the field. Square symbols in Fig. 4b mark drying-to-wetting transition points $(\sigma=0)$ where $\cos \theta_{\text {eff }}$ equals zero irrespective of approximate $\gamma_{l v}$.

In the perpendicular field, due to the orientational asymmetry of water, at each wall the value of $\sigma$ will be different. This means that for field strengths which produce an average value of $\sigma=0$ in our simulation, in fact $\sigma<0$ at the positively charged wall, and $\sigma>0$ at the 
negatively charged wall. Therefore, the positive wall should be wetting, and the negative wall drying. This situation, known as a Janus interface, shows very interesting behaviour experimentally ${ }^{34}$. Our results suggest that a Janus interface can be produced by applying a perpendicular field $E_{0} \sim 0.2 \mathrm{~V} / \AA$ across the confinement, without modifying the surface itself.

\section{CONCLUDING REMARKS}

In polar solvents like water, introduction of surface charges or the presence of applied electric field is typically associated with increased wettability. The phenomenon is relatively well understood in macroscopic systems amenable to conventional experiments and continuum description of system thermodynamics. Increasing interest in nanoporous materials, micro- and nanofluidics, and trans-membrane transport invite studies of electro-wetting in materials where characteristic length-scales become comparable to molecular dimensions. At the nanoscale regime, surface effects depend on molecular events best accessible by molecular simulations. We describe the field-induced transition from strongly hydrophobic to strongly hydrophilic behavior in the simulated, hydrocarbon-like planar confinements in equilibrium with the field-free bulk phase. The crossover between hydrophobic and hydrophilic regimes is quantified in terms of surface free energies associated with liquid spreading into confinement.

In view of the increased compressibility of the confined liquid, electrostriction effects in confinement are an order of magnitude stronger than in bulk water. Surface energies and wetting free energies appear to follow qualitative predictions from macroscopic thermodynamics. Specifically, we do not reproduce the field-induced water depletion in a confinement reported ${ }^{22}$ in a recent study of electric field effects on water between graphite-like plates. Strong field is found to stabilize the liquid phase where spontaneous evaporation of water from a narrow confinement takes place in the absence of the field ${ }^{30,33}$. 
In contrast to observations in macroscopic systems, we demonstrate a pronounced effect of field direction on water affinity to the interface. In order to optimize hydrogen bonding, angle distributions of water molecules relative to the walls are biased against orientations with both hydrogen atoms pointing toward the wall. Consequently, field effects are weakened in the perpendicular field. Here, on one of the walls the field tends to orient interfacial molecules in the unfavorable direction, resulting in different wettabilities of opposing confinement walls. In view

of incomplete screening, the field effect on wetting of a nanoscale confinement increases with the width of the pore. The differences in surface free energy, attributed to field direction, however, are virtually independent of confinement width and hence comparatively more important in narrow confinements where surface molecules represent a more significant fraction of the confined liquid. Field direction and polarity is therefore an important determinant of electro-wetting effects in a nanoporous material. Conversely, for fixed field direction and freely rotating nanotubes, surface energetics will favor water-filled tube alignment with electric field suggesting a novel mechanism to address structural order in a nanomaterial through controlled molecular or supramolecular charge distribution.

\section{Acknowledgment}

We thank Jay Rasaiah for helpful discussion. This work was supported by the National Science Foundation through awards CHE-0512131 and BES-0432625, and US Department of Energy under contract DE-AC04-94AL8500. Sandia is a multiprogram laboratory operated by Sandia Corporation, a Lockheed Martin Company, for the US Department of Energy. We thank NSF (CHE-060047) and Hewlett-Packard for computational resources.

\section{Supporting Information}

Macroscopic relations. Interaction potentials. Pressure tensor calculations. Energy reduction in electric field in systems with water/vapor and water/solid surfaces. Surface tension for model 
systems of water, aqueous film, and confined water in the absence of electric field. Includes: two graphs and two tables. Available via internet at http://pubs.acs.org

\section{References:}

(1) Israelachvili, J. N. Intermolecular and Surface Forces; Academic: London, 1992.

(2) Lu, L.; Berkowitz, M. L. Journal Of Chemical Physics 2006, 124, 101101.

(3) Mugele, F.; Baret, J. C. Journal Of Physics-Condensed Matter 2005, 17, R705.

(4) Baret, J. C.; Mugele, F. Physical Review Letters 2006, 96, 016106.

(5) Krupenkin, T.; Taylor, J. A.; Kolodner, P.; Hodes, M. Bell Labs Technical Journal 2005, 10, 161.

(6) Chen, J. Y.; Kutana, A.; Collier, C. P.; Giapis, K. P. Science 2005, 310, 1480.

(7) Berge, B.; Peseux, J. European Physical Journal E 2000, 3, 159.

(8) Krupenkin, T.; Yang, S.; Mach, P. Applied Physics Letters 2003, 82, 316.

(9) Holt, J. K.; Park, H. G.; Wang, Y. M.; Stadermann, M.; Artyukhin, A. B.; Grigoropoulos, C. P.; Noy, A.; Bakajin, O. Science 2006, 312, 1034.

(10) Jayaraman, K.; Okamoto, K.; Son, S. J.; Luckett, C.; Gopalani, A. H.; Lee, S. B.; English, D. S. Journal Of The American Chemical Society 2005, 127, 17385.

(11) Lee, J.; Moon, H.; Fowler, J.; Schoellhammer, T.; Kim, C. J. Sensors And Actuators A-Physical 2002, 95, 259.

(12) Mugele, F.; Klingner, A.; Buehrle, J.; Steinhauser, D.; Herminghaus, S. Journal Of PhysicsCondensed Matter 2005, 17, S559.

(13) Dzubiella, J.; Allen, R. J.; Hansen, J. P. Journal Of Chemical Physics 2004, 120, 5001.

(14) Lee, C. Y.; McCammon, J. A.; Rossky, P. J. Journal Of Chemical Physics 1984, 80, 4448.

(15) Shelley, J. C.; Patey, G. N. Molecular Physics 1996, 88, 385.

(16) Bateni, A.; Laughton, S.; Tavana, H.; Susnar, S. S.; Amirfazli, A.; Neumann, A. W. Journal Of Colloid And Interface Science 2005, 283, 215.

(17) Berkowitz, M. L.; Bostick, D. L.; Pandit, S. Chemical Reviews 2006, 106, 1527.

(18) Philippsen, A.; Im, W. P.; Engel, A.; Schirmer, T.; Roux, B.; Muller, D. J. Biophysical Journal 2002, 82, 1667.

(19) Dzubiella, J.; Hansen, J. P. Journal Of Chemical Physics 2004, 121, 5514.

(20) Dzubiella, J.; Hansen, J. P. Journal Of Chemical Physics 2005, 122, 234706.

(21) Vaitheeswaran, S.; Rasaiah, J. C.; Hummer, G. Journal Of Chemical Physics 2004, 121, 7955.

(22) Vaitheeswaran, S.; Yin, H.; Rasaiah, J. C. Journal Of Physical Chemistry B 2005, 109, 6629.

(23) Dzubiella, J.; Hansen, J. P. Journal Of Chemical Physics 2003, 119, 12049.

(24) Svishchev, I. M.; Kusalik, P. G. Journal Of The American Chemical Society 1996, 118, 649.

(25) Du, Q.; Freysz, E.; Shen, Y. R. Science 1994, 264, 826.

(26) Freysz, E.; Du, Q.; Shen, Y. R. Annales De Physique 1994, 19, 95.

(27) Luzar, A.; Svetina, S.; Zeks, B. Journal Of Chemical Physics 1985, 82, 5146.

(28) Lee, S. H.; Rossky, P. J. Journal Of Chemical Physics 1994, 100, 3334.

(29) Du, Q.; Superfine, R.; Freysz, E.; Shen, Y. R. Physical Review Letters 1993, 70, 2313.

(30) Leung, K.; Luzar, A.; Bratko, D. Physical Review Letters 2003, 90, 065502.

(31) Luzar, A.; Bratko, D.; Blum, L. Journal Of Chemical Physics 1987, 86, 2955.

(32) Lum, K.; Luzar, A. Physical Review E 1997, 56, R6283.

(33) Bratko, D.; Curtis, R. A.; Blanch, H. W.; Prausnitz, J. M. Journal Of Chemical Physics 2001, $115,3873$.

(34) Zhang, X. Y.; Zhu, Y. X.; Granick, S. Science 2002, 295, 663.

(35) Berendsen, H. J. C.; Grigera, J. R.; Straatsma, T. P. Journal Of Physical Chemistry 1987, 91, 6269.

(36) Truskett, T. M.; Debenedetti, P. G.; Torquato, S. Journal Of Chemical Physics 2001, 114, 2401. 
Luzar, A.; Leung, K. Journal Of Chemical Physics 2000, 113, 5836.

(38) Luzar, A. Journal Of Physical Chemistry B 2004, 108, 19859.

(39) Evans, R.; Parry, A. O. Journal Of Physics-Condensed Matter 1990, 2, SA15.

(40) Svishchev, I. M.; Kusalik, P. G. Physical Review Letters 1994, 73, 975.

(41) Suresh, S. J.; Satish, A. V.; Choudhary, A. Journal Of Chemical Physics 2006, 124, 074506.

(42) Zangi, R.; Mark, A. E. Journal Of Chemical Physics 2004, 120, 7123.

(43) Ballenegger, V.; Hansen, J. P. Journal Of Chemical Physics 2005, 122, 114711.

(44) Yang, K. L.; Yiacoumi, S.; Tsouris, C. Journal Of Chemical Physics 2002, 117, 337.

(45) Rick, S. W.; Stuart, S. J.; Berne, B. J. Journal Of Chemical Physics 1994, 101, 6141.

(46) Luzar, A.; Bratko, D. Journal Of Physical Chemistry B 2005, 109, 22545.

(47) Werder, T.; Walther, J. H.; Jaffe, R. L.; Halicioglu, T.; Koumoutsakos, P. Journal Of Physical Chemistry B 2003, 107, 1345.

(48) Arnold, A.; Holm, C. Computer Physics Communications 2002, 148, 327.

(49) Yeh, I. C.; Berkowitz, M. L. Journal Of Chemical Physics 1999, 111, 3155.

(50) Fig. $4 \mathrm{c}$ of ref. ${ }^{33}$ shows over an order of magnitude increased compressibility of interfacial water using the SPC water force field ${ }^{51}$ in a model hydrocarbon confinement. Similar results have been obtained recently using the $\mathrm{SPC} / \mathrm{E}^{35}$ water potential ${ }^{52}$.

(51) Berendsen, H. J. C.; Postma, J. P. M.; van Gunsteren, W. F.; Hermans, J. in Intermolecular Forces, Edited by B. Pullman; Reidel: Dordrecht, 1981.

(52) Giovambattista, N.; Rossky, P. J.; Debenedetti, P. G. Physical Review E 2006, 73, 041604.

(53) Sources. of different behaviors observed in ref. ${ }^{22}$ and our study may include the function ${ }^{22}$ of the barostat algorithm in a system where pressure is strongly inhomogeneous; inclusion of Ewald sums in ref. ${ }^{22}$, compared to the spherical cutoff used in the present work, seems unlikely to contribute to a qualitatively different response to the field.

(54) Kornyshev, A. A.; Sutmann, G. Physical Review Letters 1997, 79, 3435.

(55) Sutmann, G. Journal Of Electroanalytical Chemistry 1998, 450, 289.

(56) Yeh, I. C.; Berkowitz, M. L. Journal Of Chemical Physics 1999, 110, 7935.

(57) Zangi, R.; Mark, A. E. Physical Review Letters 2003, 91, 025502.

(58) Zangi, R.; Mark, A. E. Journal Of Chemical Physics 2003, 119, 1694.

(59) Zhang, X. Y.; Zhu, Y. X.; Granick, S. Journal Of The American Chemical Society 2001, 123, 6736.

(60) While. a useful measure of surface energetics, thermodynamic contact angle can differ from the geometric contact angle in a nanopore under the influence of the field. As discussed in ref. 12, the microscopic contact angle starts out at the unperturbed Young value at solid/liquid contact and will not converge to the macroscopic value over the short length separating nanopore walls.

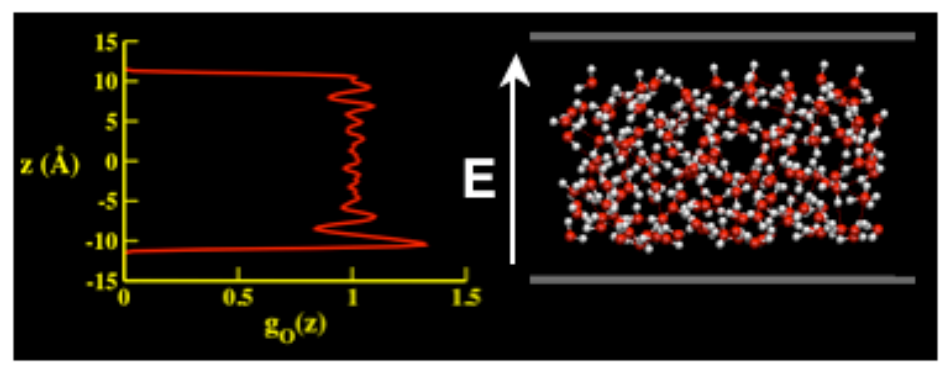

T.O.C. Figure 